\title{
A Data Mining Approach for Compressed Medical Image Retrieval
}

\author{
Vamsidhar Enireddy \\ Research scholar \\ Department of CSE \\ JNTU Kakinada
}

\author{
Kiran Kumar Reddi \\ Phd, Assistant Professor, \\ Department of Computer Science, \\ Krishna University \\ Machilipatnam
}

\begin{abstract}
The digital medical images are stored in large databases for easy accessibility and Content based image retrieval (CBIR) is used to retrieve diagnostic cases similar to the query medical image. Image compression condense the amount of data required to represent an image, it reduces the storage and transmission requirements. The medical image retrieval problem for compressed images is studied in this paper. The proposed method integrates image retrieval to retrieve diagnostic cases similar to the query medical image and image compression techniques to minimize the bandwidth utilization. Haar wavelet is used for image compression without losses. Edge and texture features are extracted from the medical compressed medical images using Sobel edge detector and Gabor transforms respectively. The classification accuracy of retrieval is evaluated using Naïve Bayes and Support Vector Machine.
\end{abstract}

\section{General Terms}

Image retrieval, Image Compression, Data Mining, Algorithm.

\section{Keywords}

Medical Images, Haar Wavelet, Sobel Edge detector, Gabor filter, Support Vector Machine, Naïve Bayes.

\section{INTRODUCTION}

With the development of medical technologies, digital images such as X-rays, MRI, ECG, CT has become a norm for diagnosis and treatment. The digital medical images are stored in large databases for easy accessibility and Content based image retrieval (CBIR) is used to retrieve diagnostic cases similar to the query medical image [1] [2]. Conventional method of image retrieval using index or semantics is not feasible as the databases contain a huge amount of data and also the image content is more versatile than the semantics. CBIR uses algorithms to extract relevant features from the image, on presenting a query image; CBIR retrieves images from the database based on the features. Features such as color, texture, edge and shape in the image are automatically extracted by CBIR systems. Similarity measures are used to compare the features extracted from the query image with that of features of images stored in the database. Images with features similar to that of query are retrieved based on some distance metric. CBIR is now widely applied in medical image applications; many CBIR systems are reviewed in literature [3] [4].
The huge quantities of diagnostic data in form of medical images are required to be stored presents a problem, and also efficient transmitting in limited bandwidth is another challenge faced. These issues are addressed by image compression. Image compression condense the amount of data required to represent an image, it reduces the storage and transmission requirements [5]. During compression process, redundancies in the image are removed resulting in compact representation of the image. Compression process is of two types: lossless compression and lossy compression. In lossless compression, the original image is perfectly recovered without any loss in the pixel value whereas in lossy compression minor loss in details occurs when image is recovered. The major advantage of lossy compression is high compression ratio is achieved.

Compression of medical images is widely studied. Medical image compression cannot afford to lose any details on recovering of image, as it may lead to problems to loss of information in diagnostically important region. Thus, compression ratio achieved through lossless compression for medical images is very less. A commonly used approach to overcome this issue is to segment the medical image into region of interest (ROI), and ROI is compressed using lossless compression and rest of the image i.e., non-ROI is compressed using lossy compression. Thus, achieve a better compression ratio while preserving quality of diagnostically crucial region.

In this paper, to retrieve diagnostic cases similar to the query medical image which are compressed to minimize the bandwidth utilization is investigated. Haar wavelet is used for image compression with a decomposition level of one to reduce the losses. Edge and texture features are extracted from the medical compressed medical images using Sobel edge detector and Gabor transforms respectively. The classification accuracy of retrieval is evaluated using Naïve Bayes and Support Vector Machine.

\section{RELATED WORK}

Liu, et al., [6] developed a novel texture feature, on the basis of Support Vector Machines (SVM). The feature was termed texture correlogram, and is used for high-level image classification. Initially, to solve the drawback of binary classification, the frame of SVM classifier is used. A novel technique was developed using hierarchical structure to deal with multiple classes from the training dataset. To help to attain the spatial distribution information, the texture correlogram is framed. For high-level image classification task, the proposed classification method and texture features are more efficient and effective than the other existing methods which are revealed from the experimental results. 
The proposed method attains better classification accuracy. The additional benefit of using the proposed method is the hierarchical structure of the SVM classification tree shows the interclass relationships between various classes. This can be used for further exploration in the relationships among highlevel concepts.

Gosselin, et al., [7] compared the classification tasks of two organizer methods. To discriminate data in the first method, the one-class and two-class SVM are used. On the basis of Gaussian Mixture the classes are modeled in the second method. As the categories searched by users are not essentially structured, better adaptation to the CBIR context is discriminative classifiers such as SVM. An adaptation is proposed concerning the specificity of the CBIR classification. The experimental tests were performed on a common database. The application of kernel in the algorithm helps to tune a specific database feature vectors easily. The kernel-based techniques are preferred in this context due to the complexity of EM. The benefits and limitations for each technique are discussed.

Mueen, et al., [8] proposed a novel image classification technique that uses multi-level image features and progressive machine learning technique, SVM. In medical image classification the existing methods manages to unite various local features or global features that are utilized separately. In the proposed method, three levels of features global (i.e., the different medical images of chest hand $\mathrm{x}$-ray images has significant difference in their structure and gray scale contrast), local (to differentiate between various organs and body parts requires regional features) and pixel (it provides excellent classification results in medical domain) are obtained and are united together in a whole (one) big feature vector. Experimental testing of the proposed method is performed to validate its efficiency. The recognition rate attained by the proposed method combined feature vector is 89\%. The Principal Component Analysis (PCA) helps to decrease the large dimensional feature vector. The proposed method was also compared with the performance of two classifiers $K$-Nearest Neighbor $(K$-NN) and Support Vector Machine (SVM).

$\mathrm{Li}$, et al., [9] proposed a novel machine learning method called multi training SVM (MTSVM). In content-based image retrieval (CBIR), the relevance feedback (RF) on the basis of support vector machines (SVMs) is used widely. But, mostly a deprived performance of SVM-based RF scheme is observed due to the number of labeled feedback samples is little. This occurrence is because of 1) the small-size training sets possess optimal hyper plane which is very perceptive to the training exemplars that is the cause for instability in the SVM classifier; and 2) due to the feature dimension being more bigger than the training sample's size, hence, the kernel technique is also not effective. The proposed MTSVM merges the advantages of co-training method and a random sampling technique in the feature space. The problems mentioned above can be lessened by the proposed MTSVM method. Experiments were conducted on a huge set of about 20000 images to validate the proposed method. The results reveal that the method developed increases the performance greater than that of the conventional SVM-based RFs in a constant manner in terms of standard deviation and precision that are implemented to examine the robustness and effectiveness of a RF method, respectively.

Chen, et al., [10] increased the retrieval performance of the content-based image retrieval by the relevance feedback methods using linear/quadratic estimators. To evaluate the sustain of target images in the excessive dimensional feature space involving a considerably less number of training samples is the main problem faced in using relevance feedback. The proposed method is based on the one-class SVM in the nonlinearly transformed feature space which suits a tight hyper-sphere based on the positive exemplars to involve the main target images. To deal the nonlinearity in the allocation of the target images in a neat way the kernel is used and better generalization ability is supported by the regularization term in SVM. The proposed method is tested on both on real-world images and synthesized data for authenticating purpose. In both cases, promising results are obtained and hence, the proposed method is advantageous to tune the parameters like the use of the Gaussian and the potency of regularization term.

\section{METHODOLOGY}

\subsection{Haar Wavelet}

The Haar Transform (HT) transforms the image from the space domain to a local frequency domain [11-12].

The Haar wavelet's mother wavelet function $\psi(t)$ can be given as:

$$
\psi(t)= \begin{cases}1 & 0 \leq t<1 / 2 \\ -1 & 1 / 2 \leq t<1, \\ 0 & \text { otherwise }\end{cases}
$$

Scaling function $\varphi(t)$ can be described as:

$$
\varphi(t)=\left\{\begin{array}{cc}
1 & 0 \leq t<1, \\
0 & \text { otherwise }
\end{array}\right.
$$

On decomposition of the signal by Hilbert transform, two components average (approximation) and detail (fluctuation) are obtained. A signal having $2^{n}$ sample values, the first average sub signal $a^{1}=\left(a_{1}, a_{2}, \ldots a_{N / 2}\right)$ for a signal length of $\mathrm{N}$ is given as:

$$
a_{n}=\frac{x_{2 n-1}+x_{2 n}}{\sqrt{2}}, n=1,2, \ldots, N / 2
$$

And the first detail sub signal $d^{1}=\left(d_{1}, d_{2}, \ldots ., d_{N / 2}\right)$ is given as:

$$
d_{n}=\frac{x_{2 n-1}-x_{2 n}}{\sqrt{2}}, n=1,2, \ldots, N / 2
$$

The transform is applied to all rows of the matrix. A resultant matrix of first level is formed by placing the approximation parts of each row transform in the first $n$ columns and the corresponding detail parts in the last $\mathrm{n}$ columns. The resultant matrix has four pieces with each piece of dimension (number of rows/2) X (number of columns/2). Each piece is termed $A$, $H, V$ and $D . A$ is the approximation area, $\mathrm{H}$ is the horizontal area, $\mathrm{V}$ is vertical area and $\mathrm{D}$ is diagonal area. 


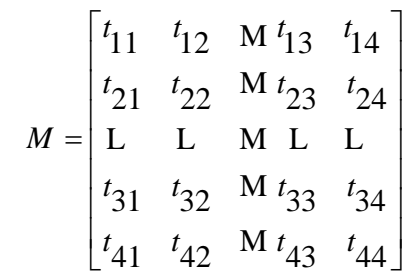

$$
\begin{aligned}
& A=\left(\begin{array}{ll}
t_{11} & t_{12} \\
t_{21} & t_{22}
\end{array}\right) \quad H=\left(\begin{array}{ll}
t_{13} & t_{14} \\
t_{23} & t_{24}
\end{array}\right) \\
& V=\left(\begin{array}{ll}
t_{31} & t_{32} \\
t_{41} & t_{42}
\end{array}\right) \quad D=\left(\begin{array}{ll}
t_{43} & t_{44} \\
t_{53} & t_{54}
\end{array}\right)
\end{aligned}
$$

\subsection{Sobel Edge Detector}

Edge detection is the process of identifying and locating sharp discontinuities in an image, which are abrupt changes in pixel intensity characterizing boundaries of objects in a scene. Classical edge detection methods involve convolving image with an operator (a 2-D h filter), sensitive to the image's large gradients while returning zero value in uniform regions. Many edge detection operators are designed to be sensitive to certain edge types. Variables involved in edge detection operator selection include Edge orientation, Noise environment and Edge structure. The operator's geometry determines the direction where it is very sensitive to edges. Operators are trained to look for horizontal, vertical, or diagonal edges. Edge detection is tough in noisy images, as both noise and edges have high frequency content. Attempts to reduce noise lead to blurred/distorted edges. Operators on noisy images are larger and so they average enough data to discredit localized noisy pixels. This leads to less accurate localization of edge detection. All edges do not need an intensity step change.

The Sobel Edge Detector generates a series of gradient magnitudes with a simple convolution kernel. Applying convolution $K$ to pixel group $p$ can be represented as:

$$
N(x, y)=\sum_{k=-1}^{1} \sum_{j=-1}^{1} K(j, k) p(x-j, y-k)
$$

The Sobel edge detector consists of a pair of $3 \times 3$ convolution kernels as shown in Figure 1. One kernel is just the other rotated by $90^{\circ}$.

\begin{tabular}{|l|l|l|}
\hline-1 & 0 & +1 \\
\hline-2 & 0 & +2 \\
\hline-1 & 0 & +1 \\
\hline \multicolumn{4}{|c|}{$\mathrm{Gx}$}
\end{tabular}

\begin{tabular}{|l|l|l|}
\hline+1 & +2 & +1 \\
\hline 0 & 0 & 0 \\
\hline-1 & -2 & -1 \\
\hline \multicolumn{3}{c|}{ Gy }
\end{tabular}

\section{Figure 1: Convolution kernels of Sobel detector}

The kernels respond maximally to vertically and horizontally running edges, relative to a pixel grid, one kernel for each of the two perpendicular orientations. The kernels are applied separately to input image, producing separate gradient component measurements in each orientation. One detecting changes in vertical contrast $\left(G_{x}\right)$ and the other detecting horizontal contrast $\left(G_{y}\right)$, which can then be combined together to seek the absolute gradient magnitude at every point and its orientation [13].

The two gradients computed using $\mathrm{G}_{\mathrm{x}}$ and $\mathrm{G}_{\mathrm{y}}$ can be regarded as the $\mathrm{x}$ and $\mathrm{y}$ components of the vector. They therefore have a gradient magnitude and direction:

$$
\begin{aligned}
& g=\left[\begin{array}{l}
g_{x} \\
g_{y}
\end{array}\right] \\
& g=\sqrt{g_{x}^{2}+g_{y}^{2}} \\
& \theta=\tan ^{-1}\left(\frac{g_{y}}{g_{x}}\right)
\end{aligned}
$$

where $\mathbf{g}$ is the gradient vector and $\mathbf{g}$ the gradient magnitude.

\subsection{Gabor Filter}

Gabor filters were widely used as a texture model for image interpretation tasks as there are strong relations between different filters outputs. These relationships devise a new texture feature capable of describing texture information in a concisely manner. Information on filter distribution responses is encoded in the new feature. Feature performance is assessed through application to an image region classification task and comparing results with those obtained with features that do not utilize filter outputs relationships. Encoding textural information into a feature vector for use in image understanding tasks is a major issue. First order statistics, are severely limited. Visually different Image regions can have the same first order statistics. Use of second order statistics enables improvement of the situation taking into account not just grey pixel levels but also spatial relationships between them [14]. Gabor filter, Gabor transform are widely applied to image processing, computer vision and pattern recognition. This function can provide accurate time-frequency location governed by the "Uncertainty Principle" [15].

A two dimensional Gabor function $\mathrm{g}(\mathrm{x}, \mathrm{y})$ and its Fourier transform $\mathrm{G}(\mathrm{u}, \mathrm{v})$ is given by:

$$
\begin{aligned}
& g(x, y)=\left(\frac{1}{2 \pi \sigma_{x} \sigma_{y}}\right) \exp \left[-\frac{1}{2}\left(\frac{x^{2}}{\sigma_{x}^{2}}+\frac{y^{2}}{\sigma_{y}^{2}}\right)+2 \pi j W x\right] \\
& G(u, v)=\exp \left\{-\frac{1}{2}\left[\frac{(u-W)^{2}}{\sigma_{u}^{2}}+\frac{v^{2}}{\sigma_{v}^{2}}\right]\right\}
\end{aligned}
$$

Where $\sigma_{u}=1 / 2 \pi \sigma_{x}$ and $\sigma_{v}=1 / 2 \pi \sigma_{y}$.

\subsection{Naïve Bayes}

The Naïve Bayes is a probabilistic method used for prediction. During training the conditional probabilities of each attribute in the predicted class is estimated from the training data set. The parameter's mean and variance obtained from a small set of training data is sufficient for classification. Commonly used as the Naïve Bayes provide good results and easy interpretation of the results [16]. The disadvantage being that the classifier considers that the occurrence of attributes is 
independent; therefore the correlation between the attributes is ignored.

Naïve Bayes classifies the given input represented by its feature vector to the most likely class. Learning is simplified on the assumption that the features are independent given class,

$$
P(X \mid C)=\prod_{i=1}^{n} P\left(X_{i} \mid C\right)
$$

Where $\mathrm{X}=\left(\mathrm{X}_{1}, \ldots, \mathrm{X}_{\mathrm{n}}\right)$ is the feature vector and $\mathrm{C}$ is a class.

\subsection{Support Vector Machine (SVM)}

Support Vector Machines (SVM's) are a new learning method for binary classification, where basic idea is to locate a hyperplane that separates d-dimensional data into its two classes perfectly [17]. But as example data is usually not linearly separable, SVM's provide the idea of a kernel induced feature space that casts data into a higher dimensional space where it is separable. Casting into such space can cause computational problems, and also the issue with overfitting. A key insight in SVM's is that higher-dimensional space does not need to be handled directly (only formula for dot-product in such space is needed), which in turn eliminates above concerns. Further, VC-dimension (a measure of a system to perform well on unseen data) of SVM's can be correctly calculated, unlike other methods like neural networks which have no measure. Altogether, SVM's are intuitive, wellfounded theoretically, and are practically successful. SVM's have also solved regression tasks (where systems are trained to output numerical values and not a lyes/no" classification).

The aim of SVM is production of a model (based on training data) that predicts target values of test data when given only test data attributes. Given a training set of instance-label pairs (xi; yi); i $=1 ;::: ; 1$, the support vector machines (SVM) need a solution of the following optimization problem [18]:

$$
\min _{w, b, \xi} \frac{1}{2} w^{T} w+C \sum_{i=1}^{l} \xi_{i}
$$

Subject to

$y_{i}\left(w^{T} \phi\left(x_{i}\right)+b\right) \geq 1-\xi_{i}$,

$\xi_{i} \geq 0$

A classification task usually involves with training and testing data which consist of some data instances. Each instance in the training set contains one "target value" (class labels) and several "attributes" (features). The goal of SVM is to produce a model which predicts target value of data instances in the testing set which are given only the attributes. A classification problem is restricted to consideration of a two-class problem without loss of generality [19].

\section{RESULTS AND DISCUSSION}

The Haar wavelet is used for compressing the medical images. The edge and texture features from the images are extracted using Sobel edge detector and Gabor transforms respectively for image retrieval. The classification accuracy of retrieval is evaluated using Naïve Bayes and Support Vector Machine.
Table 1 tabulates the classification accuracy and RMSE, and Figure 2 shows the same.

Table 1: Classification Accuracy

\begin{tabular}{|l|l|l|}
\hline Technique & $\begin{array}{l}\text { Classification } \\
\text { Accuracy }\end{array}$ & RMSE \\
\hline Naïve Bayes & $92 \%$ & 0.2828 \\
\hline C-SVM with linear kernel & $91 \%$ & 0.3 \\
\hline $\begin{array}{l}\text { nu-SVM with linear } \\
\text { kernel }\end{array}$ & $92 \%$ & 0.2828 \\
\hline
\end{tabular}

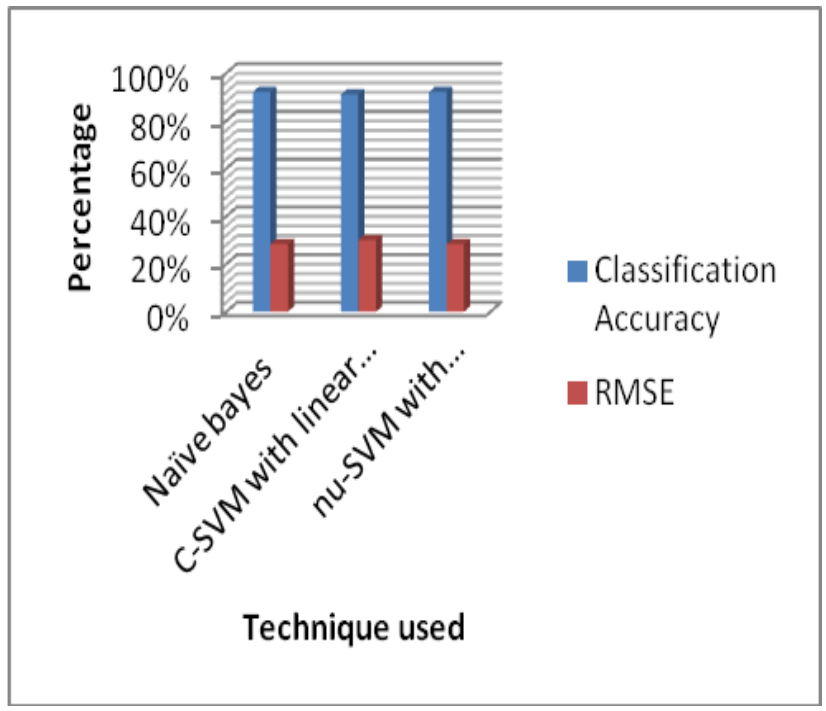

Figure 2: Classification accuracy and RMSE for different techniques

Table 2 lists the precision, recall and $\mathrm{f}$ Measure for various classification techniques. Figure 3 shows the precision and recall, and Figure 4 shows the f measure.

Table 2: Precision, Recall and F Measure

\begin{tabular}{|l|l|l|l|}
\hline Technique & Precision & Recall & $\begin{array}{l}\text { F- } \\
\text { Measure }\end{array}$ \\
\hline Naïve Bayes & 0.92 & 0.92 & 0.92 \\
\hline $\begin{array}{l}\text { C-SVM with linear } \\
\text { kernel }\end{array}$ & 0.91 & 0.91 & 0.91 \\
\hline $\begin{array}{l}\text { nu-SVM with linear } \\
\text { kernel }\end{array}$ & 0.92 & 0.92 & 0.92 \\
\hline
\end{tabular}




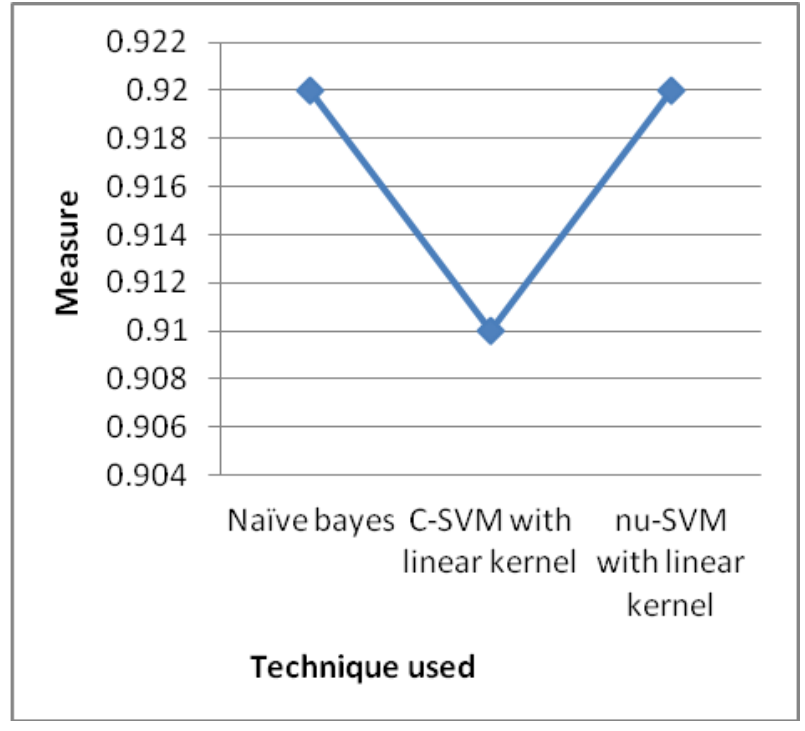

Figure 3:Measured Precision

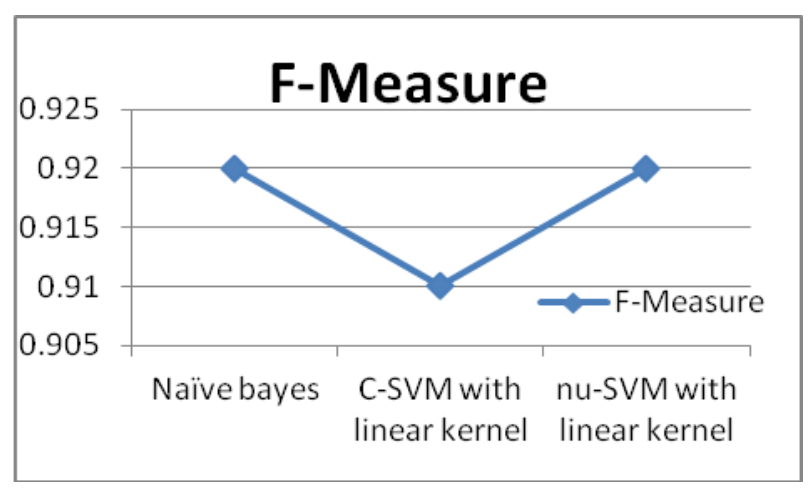

Figure 4: $f$ Measure

\section{CONCLUSION}

This paper proposed to investigate the Image Retrieval (IR) problem on compressed images. Traditional techniques proposed in literature extensively investigated feature extraction on uncompressed images. The classification accuracy obtained is comparable to the accuracies obtained in uncompressed images. Further work needs to be carried out to investigate the effectiveness of soft computing classification algorithms for compressed medical image retrieval.

\section{REFERENCES}

[1] Lehmann, T.M., Schubert, H. , Keysers, D., Kohnen, M., Wein, B.B , The IRMA code for unique classification of medical image, in the Proceedings of the SPIE 5033, 109-117 (2003).

[2] Samuel, G., Armato III, et al.: Lung image database consortium - Developing a resource for the medical imaging research community, in Radiology . 232, 739748 (2004).

[3] Crucianu M., Ferecatu M., Boujemaa N.: Relevance vthe Art in Audiovisual Content-Based Retrieval, Information Universal Access and Interaction, 2004.
[4] Muller, H., Michoux, N., Bandon, D., Geissbuhler, A, A review of content based image retrieval systems in medical applications - Clinical benefits and future directions, in the International Journal of Medical Informatics .73, 1-23 (2004).

[5] Cerra, D. ,Datcu, M., Image Retrieval using Compression based techniques, in Proceedings of the International Conference on Source and Channel Coding (SCC), 1-6 (2010)

[6] S. Liu, H. Yi, L.-T. Chia, and D. Rajan, "Adaptive Hierarchical Multiclass SVM classifier for Texture-based Image Classification," in Proceedings of ICME, pp. 1190-1193, 2005.

[7] P. Gosselin, M. Najjar, M. Cord, and C. Ambroise. Discriminative classification vs modeling methods in CBIR. In IEEE Advanced Concepts for Intelligent Vision Systems (ACIVS), Brussel, Belgium, September 2004.

[8] A. Mueen, M. Sapian Baba, and R. Zainuddin. Multilevel feature extraction and x-ray image classification. J. Applied Sciences, 7(8):1224-1229, 2007.

[9] Li, J., Allinson, N., Tao, D., And Li, X. 2006. Multitraining support vector machine for image retrieval. IEEE Transactions on Image Processing 15, 11, 35973601 .

[10] Y. Chen, X. Zhou, and T.S. Huang, "One-Class SVM for Learning in Image Retrieval," Proc. IEEE Int'l Conf. Image Processing, pp. 815-818, 2001.

[11] A.Haar, in Zur Theorie der orthogonalen Funktionen systeme. Mathematics Annual pp 331-371, 1910.

[12] Kamrul Hasan Talukder, Koichi Harada , Haar Wavelet Based Approach for Image Compression and Quality Assessment of Compressed Image, in IAENG International Journal of Applied Mathematics, pp 49-56, 2007.

[13] Raman Maini \& Dr. Himanshu Aggarwal ," Study and Comparison of Various Image Edge Detection Techniques ",International Journal of Image Processing (IJIP), Volume (3) : Issue (1), pp. 1 - 12

[14] C.K. Chui, An Introduction to Wavelets, Academic Press, Boston, 1992

[15] C J Setchell, N W Campbell ,'Using Colour Gabor Texture Features For Scene Understanding." In Proc. $7^{\text {th }}$ Internat Conf. on image processing applications. Vol. 67(5), pp. 372-376.

[16] Besserve. M, Garnero. L, Martinerie. J. Cross-Spectral Discriminant Analysis (CSDA) for the classification of Brain Computer Interfaces. 3rd International IEEE/EMBS Conference on Neural Engineering, 2007. CNE '07. pp:375 - 378,2007

[17] Dustin Boswell, 2002,'Introduction to Support Vector Machines"

[18] Chih-Wei Hsu, Chih-Chung Chang, and Chih-Jen Lin, 2010,"A Practical Guide to Support Vector Classification"

[19] Steve R. Gunn, 1998,"Support Vector Machines for Classification and Regression. 